\title{
A Rare Presentation of Acquired Laryngomalacia and Tracheomalacia in a Child Associated with Apricot Sulfurization
}

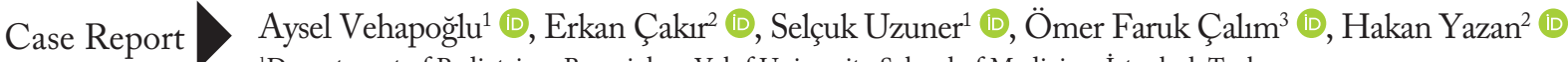 \\ ${ }^{1}$ Department of Pediatrics, Bezmialem Vakıf University School of Medicine, İstanbul, Turkey \\ ${ }^{2}$ Department of Pediatric Pulmonology, Bezmialem Vakıf University School of Medicine, İstanbul, Turkey \\ ${ }^{3}$ Department of Otorhinolaryngology, Bezmialem Vakıf University School of Medicine, İstanbul, Turkey
}

\begin{abstract}
Sulfur fumigation has come to replace traditional sun drying methods for drying fruits over the years around the world as it is a cheaper and faster method because of its pesticidal and anti-bacterial properties. We report the case of an 11-year-old boy with acquired severe biphasic stridor who was exposed to extremely high concentrations of sulfur dioxide (SO2) during apricot sulfurization processes with his mother. The patient's bronchoscopy revealed severe glottic and subglottic damage. Exposure to $\mathrm{SO} 2$ is a health risk, particularly for individuals who are sulfide-sensi-
\end{abstract}

ORCID iDs of the authors: A.V. 0000-0002-4000-6330: E.C. 0000-0002-1438-7854; S.U. 0000-0002-7133-5452; O.F.C. 0000-0002-0010-9028; H.Y. 0000-0002-7680-4000.

Cite this article as: Vehapoğlu A, Çakır E, Uzuner S, çalım ÖF, Yazan H. A Rare Presentation of Acquired Laryngomalacia and Tracheomalacia in a Child Associated with Apricot Sulfurization. Turk Arch Otorhinolaryngol 2020; 58(4):286-8.

\section{Corresponding Author:}

Aysel Vehapoğlu; ayvahap@hotmail.com

Received Date: 15.04 .2020

Accepted Date: 01.09 .2020

Content of this journal is licensed under a Creative Commons Attribution 4.0 International License. Available online at www.turkarchotolaryngol.net

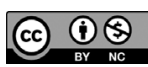

DOl: 10.5152/tao.2020.5398

\section{Introduction}

Stridor is one of the physical signs of upper airway obstruction. It is a vibrating sound produced when the airway is obstructed, arising from turbulent airflow in the respiratory passages (1). Stridor can be inspiratory, expiratory or biphasic. Commonly, inspiratory stridor suggests an obstruction above the glottis owing to the collapse of soft the tissues, and expiratory stridor suggests an obstruction in the lower trachea. Biphasic stridor typically results from lesions at the level of the glottis or subglottis (2).

Inhaled toxic substances may directly damage the pulmonary epithelium at the various levels of the respiratory system. One of these substances is sulfur dioxide (SO2) which has many industrial and agricultural uses. It is a colorless toxic gas and one of the most hazardous air pollutants (3). Sulfur fumigation, which has been used for many years, has pesticide and anti-bacterial properties. It is increasingly used in place of the traditional sun drying technique for fruits and vegetables because it is cheaper, faster, and easier (4). Inhalation of high concentrations of $\mathrm{SO} 2$ primarily affects the upper respiratory tract and the lungs and can have immediate life-threatening effects (5). Gastrointestinal symptoms (such as nausea, vomiting and diarrhea) have been clinically reported. Most reactions to sulfites are characterized by severe tive, especially in childhood. The pulmonary epithelium may be directly injured by inhaled toxic substances at various levels of the respiratory system. To the best of our knowledge, this is the first case reported of acquired airway damage associated with sulfurization in a pediatric patient without a known history of any respiratory disease or symptoms.

Keywords: Sulfur dioxide, laryngomalacia, tracheomalacia, pediatrics, tracheotomy, pediatric otolaryngology dyspnea, wheezing, and bronchospasm, which can occur within minutes after exposure to materials containing sulfite (6). There is, however, limited information about the effects of sulfur fumigation on herbal safety and efficacy in children. Here we describe a male child with acquired progressive biphasic stridor who inhaled extremely high concentrations of $\mathrm{SO} 2$ during apricot sulfurization process.

\section{Case Presentation}

An 11-year-old male patient presented with cough, progressive respiratory distress, and noisy breathing. His complaints had begun two months earlier, and he was given several medications with the diagnosis of reactive respiratory tract disease. The patient with ever-increasing complaints became unable to sleep at night due to respiratory distress. The child's physical examination revealed dyspnea, biphasic stridor, suprasternal retractions, and rhonchi in the lung. Initially, it was learned that the child had been continuously eating ice for more than one month. A chest X-ray revealed a long segment of stenosis in the trachea (Figure 1) and neck magnetic resonance imaging (MRI) revealed diffuse edematous intense contrast enhancement in the larynx, and an edematous image compressing the trachea and stenosis at the level of the vocal cords. Bronchoscopy performed under 
general anesthesia revealed laryngomalacia and tracheomalacia. Tracheotomy was performed to relieve deteriorating respiration. The patient was given anti-reflux treatment for two months because gastro-esophageal reflux disease is strongly associated with laryngomalacia and is one of the major causes of acquired laryngomalacia. The bronchoscopy performed two months later showed no changes in the previous findings. The epiglottis was edematous and had lost its natural structure; the arytenoid cartilage was severely edematous and obstructed the respiratory tract. The glottic and the subglottic area was seen to be narrowed with $90 \%$ edema. In the bronchoscopy performed at the 6th month of the treatment, the epiglottis and the vocal cords were seen to have near-normal appearance (Figure 2). After a 1-year follow-up, the patient was decannulated and observed to be free of problems. The patient has been followed for one year without any problems.

Written informed consent was obtained from the patient's parents for publication of this case report.

\section{Discussion}

Here we report a case of an 11-year-old male child employed in apricot sulfurization with his mother. The child who did not have a medical history of prior respiratory disease or symptoms presented with cough, severe biphasic stridor and respiratory distress. His initial bronchoscopy demonstrated acquired glottic injury, laryngomalacia, and tracheomalacia with tracheal edema. Initially, it was learned that the child had been continuously eating ice for more than one month. The detailed story of the patient revealed that the child had been working in apricot production with his mother for one month. The child was reported to have inhaled extremely high concentrations of $\mathrm{SO} 2$ during sulfurization process that lasted about one month in Malatya. Acquired severe glottis injury associated with apricot sulfurization has not been reported before.

Exposure to SO2 is a health risk, especially for individuals sensitive to sulfide. Individuals working in the apricot sulfurization process have been reported to present with respiratory symptoms such as itchy eyes, cough, runny nose, itchy throat, shortness of breath, and decreased lung function (7). SO2 inhalation leads to acute lung inflammation and respiratory tract hyperreactivity. Moreover, it has been shown that long time inhalation of sulfur dioxide increased oxidative stress and bronchial inflammation, reduced lung function, and increased the risk of lung cancer development (8). Clinicians should consider toxic gas inhalation

\section{Main Points}

- Physicians should be aware that acquired laryngomalacia and tracheomalacia in children may be due to exposure to toxic gas inhalation.

- Taking a detailed history is, as in all diseases, the most crucial approach to diagnose respiratory diseases.

- Complete and efficient evaluation and diagnosis of the stridor in children are critical for safe and timely management and intervention.

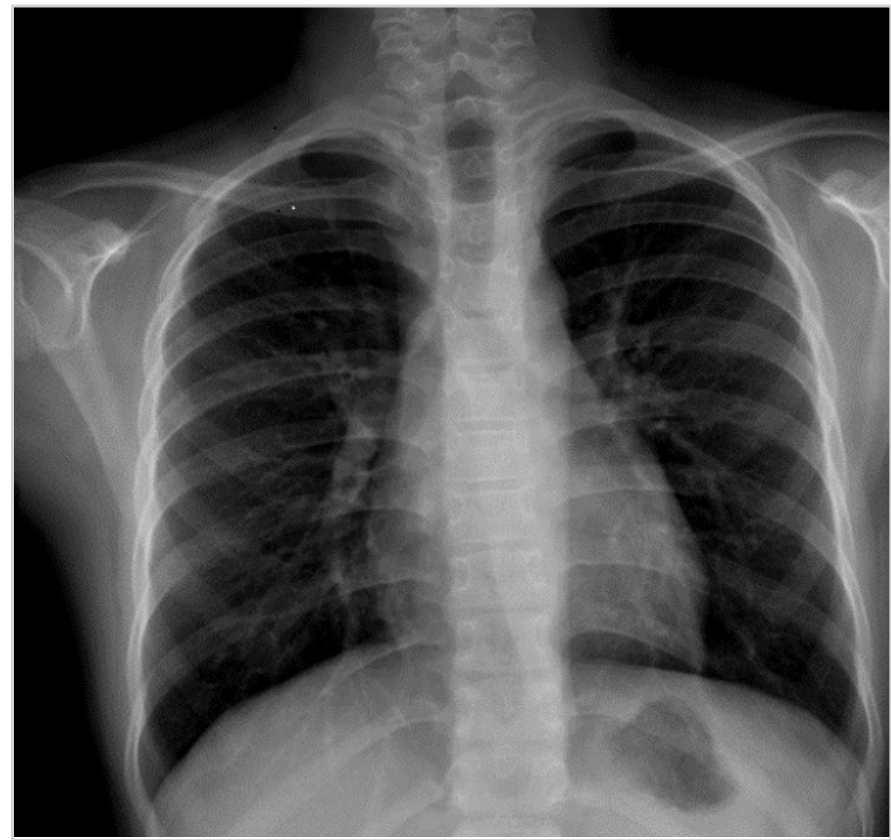

Figure 1. Chest X-ray showing a long segment of stenosis in the trachea
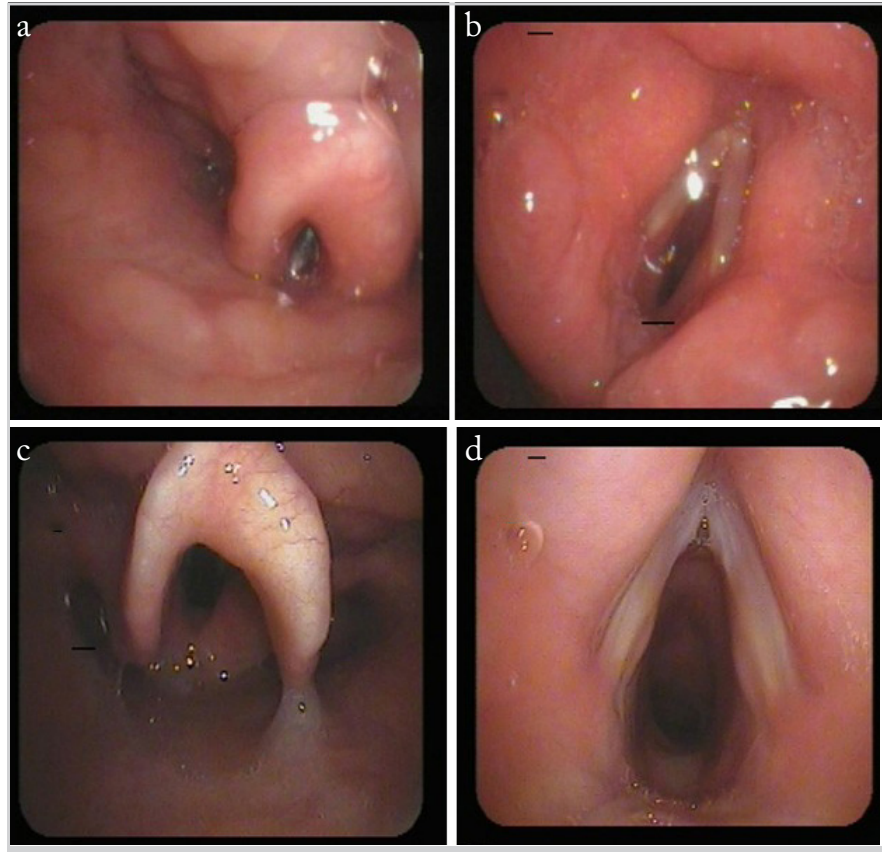

Figure 2. a-d. (a, b) Severe edema and bullous lesions in epiglottis, vocal cords and subglottic area. (c, d) Close to normal appearance on epiglottis and vocal cords after 6 months

in every patient with respiratory problems with acute onset and which do not respond to treatment.

The mechanism of $\mathrm{SO} 2$ damage to the lungs and the long-term results of this damage are not clear. Animal studies showed that inhalation of $\mathrm{SO} 2$ causes a local pulmonary inflammatory reaction and systemic effects within a few hours after exposure as an acute response (9).

Malatya, which is a city in the eastern part of Turkey, is internationally renowned as a dried apricot producing region, with 
a share of $7-10 \%$ of the world's table apricots and $80-85 \%$ of dried apricots. Sulfurization of apricots is done in July each year and the process takes about 30-35 days. Individuals working in apricot sulfurization inhale $\mathrm{SO} 2$ gas during this process (10).

Physicians should be aware that acquired laryngomalacia and tracheomalacia in children may be due to exposure to toxic gas inhalation. Taking a detailed history is, as in all diseases, the most crucial approach to diagnose respiratory diseases. Complete and efficient evaluation and diagnosis of the stridor in children are critical for safe and timely management and intervention.

\section{Conclusion}

Apricot sulfurization workers are exposed to high concentrations of $\mathrm{SO} 2$. Children are more vulnerable than adults. $\mathrm{SO} 2$ exposure can lead to respiratory problems. Symptoms of $\mathrm{SO} 2$ gas inhalation injury vary and can occur immediately after exposure or have a delayed reaction, causing injury anywhere along the respiratory tract, and the exposure may lead to inflammatory changes.

Informed Consent: Written informed consent was obtained from the parents of the patient.

Peer-review: Externally peer-reviewed.

Author Contributions: Concept - A.V.; Materials - E.Ç.; Data Collection and/or Processing - S.U., Ö.F.Ç.; Literature Search - H.Y.; Writing - A.V.

Conflict of Interest: The authors have no conflicts of interest to declare.
Financial Disclosure: The authors declared that this study has received no financial support.

\section{References}

1. Marchese A, Langhan ML. Management of airway obstruction and stridor in pediatric patients. Pediatr Emerg Med Pract 2017; 14: $1-24$

2. Darras KE, Roston AT, Yewchuk LK. Imaging acute airway obstruction in infants and children. Radiographics 2015; 35: 206479 .

3. Gorguner M, Akgun M. Acute inhalation injury. Eurasian J Med 2010; 42: 28-35.

4. Kumar A, Triquigneaux M, Madenspacher J, Ranguelova K, Bang JJ, Fessler MB, et al. Sulfite-induced protein radical formation in LPS aerosol-challenged mice: implications for sulfite sensitivity in human lung disease. Redox Biol 2018; 15: 327-34.

5. Wang XB, Du JB, Cui H. Sulfur dioxide, a double-faced molecule in mammals. Life Sci 2014; 98: 63-7.

6. Vally H, Misso NL, Madan V. Clinical effects of sulphite additives. Clin Exp Allergy 2009; 39: 1643-51.

7. Koksal N, Hasanoglu HC, Gokirmak M, Yildirim Z, Gultek A. Apricot sulfurization: an occupation that induces an asthma-like syndrome in agricultural environments. Am J Ind Med 2003; 43: 447-53.

8. Rusconi F, Catelan D, Accetta G, Peluso M, Pistelli R, Barbone F, et al. Asthma symptoms, lung function, and markers of oxidative stress and inflammation in children exposed to oil refinery pollution. J Asthma 2011; 48: 84-90.

9. Wigenstam E, Elfsmark L, Bucht A, Jonasson S. Inhaled sulfur dioxide causes pulmonary and systemic inflammation leading to fibrotic respiratory disease in a rat model of chemical-induced lung injury. Toxicology 2016; 368-369: 28-36.

10. Uren N, Yuksel S, Onal Y. Genotoxic effects of sulfur dioxide in human lymphocytes. Toxicol Ind Health 2014; 30: 311-5. 